\title{
THE IDEAL DETERMINED BY THE UNSYMMETRIC GAME
}

\author{
L. NEWELSKI AND A. ROSLANOWSKI
}

(Communicated by Andreas R. Blass)

\begin{abstract}
In the present paper we study the ideal of all subsets of $\mathscr{X}^{\omega}$ for which the second player has a winning strategy in the unsymmetric game. We describe its cardinal coefficients and the notions of forcing determined by it.
\end{abstract}

\section{INTRODUCTION}

Let $\Gamma_{\mathscr{Z}}^{*}(A)$ denote the unsymmetric game on the set $A \subseteq \mathscr{X}^{\omega}$. In this game Player I chooses finite sequences of elements of $\mathscr{X}$ while Player II chooses single elements of $\mathscr{X}$. They play alternately to construct a function $c=$ $s_{1}^{\wedge} n_{1}^{\wedge} s_{2} \wedge n_{2} \wedge \cdots \in \mathscr{Q}^{\omega}$, where $s_{i}$ is the $i$ th sequence chosen by the first player and $n_{i}$ is the answer of the second. Player I wins if and only if the result $c$ of the game belongs to $A$.

One can easily observe that the family of all $A \subseteq \mathscr{X}^{\omega}$ for which Player II has a winning strategy in $\Gamma_{\mathscr{D}}^{*}(A)$ is a $\sigma$-ideal. Denote this ideal by $\mathfrak{D}_{\mathscr{L}}$. Davis proved that if the second player had a winning strategy in $\Gamma_{\mathscr{X}}^{*}(A)$ then he had one that did not depend on the history of the game, a strategy that did not need to know who played which element (cf. [D]). Thus

$$
\begin{aligned}
\mathfrak{D}_{\mathscr{X}}=\left\{A \subseteq \mathscr{X}^{\omega}:\right. & \left(\exists f: \mathscr{X}^{<\omega} \rightarrow \mathscr{X}\right)(\forall c \in A) \\
& (\exists n \in \omega)(\forall m>n)(c(m) \neq f(c \mid m))\} .
\end{aligned}
$$

The ideal $\mathfrak{D}_{\mathscr{X}}$ appeared implicitly in Mycielski's proof of the determinacy of analytic sets in games $\Gamma_{\mathscr{Z}}^{*}$ (cf. [M]); it was studied in [R1]. In the present paper we continue those investigations.

Our notation and terminology are standard and derived from [J].

Let $\mathscr{X}$ be a countable set with at least two elements. We will think of $\mathscr{X}$ as an element of $\omega \cup\{\omega\}$.

\section{General PROPERTIES}

The space $\mathscr{X}^{\omega}$ is endowed with the product topology determined by the basis $\left\{[s]: s \in \mathscr{X}^{<\omega}\right\}$, where $[s]=\{c \in \mathscr{X} \omega: s \subseteq c\}$. If $\mathscr{X}$ is finite then the Lebesgue measure $\lambda$ on the space $\mathscr{X}^{\omega}$ is the obvious product measure. In the space $\omega^{\omega}$, $\lambda$ is the product of the measure $\lambda_{0}$ on $\omega$ defined by $\lambda_{0}(\{n\})=2^{-(n+1)}$. Let $\mathbb{K}\left(\mathscr{Z}^{\omega}\right)$ and $\mathbb{L}\left(\mathscr{Z}^{\omega}\right)$ stand for $\sigma$-ideals of first category (meager) sets of the

Received by the editors December 26, 1990 and, in revised form, July 2, 1991.

1991 Mathematics Subject Classification. Primary 04A15; Secondary 03E40, 90D13. 
space $\mathscr{X}^{\omega}$ and of Lebesgue null sets in the space $\mathscr{X}^{\omega}$. BOREL $\left(\mathscr{X}^{\omega}\right)$ is the family of all Borel subsets of $\mathscr{X}^{\omega}$. If $\mathbb{I}$ is a $\sigma$-ideal on $\mathscr{X}^{\omega}$ then BOREL(I) denotes the $\sigma$-algebra generated by BOREL $\cup \mathbb{I}$. Two ideals $\mathbb{I}$ and $\mathbb{J}$ on $\mathscr{X}^{\omega}$ are orthogonal if there exists sets $A \in \mathbb{I}$ and $B \in \mathbb{J}$ such that $A \cup B=\mathscr{X}^{\omega}$.

Recall some results concerning $\mathfrak{D}_{\mathscr{X}}$ from [R1].

Theorem 1.1. (a) $\mathfrak{D}_{\mathscr{X}}$ is a $\sigma$-ideal with a Borel basis. If $\mathscr{X}$ is a group then $\mathfrak{D}_{\mathscr{X}}$ is translation invariant and symmetric.

(b) $\mathfrak{D}_{\mathscr{X}} \subseteq \mathbb{K}\left(\mathscr{X}^{\omega}\right)$. If $\mathscr{X}$ is finite then $\mathfrak{D}_{\mathscr{X}} \subseteq \mathbb{L}\left(\mathscr{X}^{\omega}\right)$.

(c) $\mathfrak{D}_{\omega}$ is orthogonal to $\mathbb{L}\left(\omega^{\omega}\right)$ and contains all compact subsets of $\omega^{\omega}$. $\mathfrak{D}_{2}=\left[2^{\omega}\right] \leq \omega$.

(d) There exist c disjoint closed subsets of $\mathscr{X}^{\omega}$ that do not belong to $\mathfrak{D}_{\mathscr{X}}$.

(e) BOREL $\left(\mathfrak{D}_{\mathscr{Z}}\right)$ is not closed under the Souslin operation $\mathscr{A}$.

A tree on $\mathscr{X}$ is a subset $T$ of $\mathscr{X}^{<\omega}$ such that $(\forall s \in T)(\forall t \subseteq s)(t \in T)$. If $s$ is an element of a tree $T$ then $\operatorname{succ}_{T}(s)=\left\{x \in \mathscr{X}: s^{\wedge} x \in T\right\}$. The body [T] of a tree $T$ is a set of all infinite branches through $T,[T]=\left\{c \in \mathscr{X}^{\omega}:(\forall n \in\right.$ $\omega)(c \mid n \in T)\}$.

It follows that each set from $\mathfrak{D}_{\mathscr{X}}$ can be covered by the countable union of bodies of trees on $\mathscr{X}$ which satisfy

$$
(\forall s \in T)\left(\operatorname{succ}_{T}(s) \neq \mathscr{X}\right) .
$$

Clearly, $[T] \in \mathfrak{D}_{\mathscr{X}}$ provided $T$ satisfies $(*)$. On the other hand, if the first player has a winning strategy in $\Gamma_{\mathscr{Z}}^{*}(A)$ then $A$ contains the body of a tree $T$ on $\mathscr{X}$ such that

$$
(\forall s \in T)(\exists t \in T)\left(s \subseteq t \& \operatorname{succ}_{T}(t)=\mathscr{X}\right) .
$$

Obviously, Player I can win $\Gamma_{\mathscr{X}}^{*}([T])$ provided $T$ satisfies $(* *)$.

Theorem $1.2[\mathrm{M}]$. If $A \in \Sigma_{1}^{1}\left(\mathscr{X}^{\omega}\right)$ then either $A \in \mathfrak{D}_{\mathscr{X}}$ or there exists a tree $T$ on $\mathscr{X}$ such that $[T] \subseteq A$ and $T$ satisfies (**).

Proposition 1.3. (a) Suppose $\mathscr{X} \subseteq \mathscr{Y}$. Then there exists a continuous function $\phi: \mathscr{Y}^{\omega} \rightarrow \mathscr{X}^{\omega}$ such that $\phi^{-1}[A] \in \mathfrak{D}_{\mathscr{Y}}$ provided $A \in \mathfrak{D}_{\mathscr{X}}$.

(b) There exists a continuous function $\psi: \omega^{\omega} \rightarrow \omega^{\omega}$ such that $\psi^{-1}[A] \in \mathfrak{D}_{\omega}$ provided $A \in \mathbb{K}\left(\omega^{\omega}\right)$.

Proof. (a) Fix $x_{0} \in \mathscr{X}$. Let $\phi^{\prime}: \mathscr{Y}^{<\omega} \rightarrow \mathscr{X}^{<\omega}$ be defined by

$$
\phi^{\prime}(s)(n)= \begin{cases}s(n), & s(n) \in \mathscr{X}, \\ x_{0}, & s(n) \notin \mathscr{X},\end{cases}
$$

for $s \in \mathscr{Y}^{<\omega}$ and $n<\operatorname{lh}(s)$. For $c \in \mathscr{Y}^{\omega}$ put $\phi(c)=\bigcup_{n \in \omega} \phi^{\prime}(c \mid n)$. Clearly, $\phi: \mathscr{Y}^{\omega} \rightarrow \mathscr{X}^{\omega}$ is continuous. Suppose that $A \in \mathfrak{D}_{\mathscr{X}}$ and $f: \mathscr{X}^{<\omega} \rightarrow \mathscr{X}$ is a witness for it. Let $g=f \circ \phi^{\prime}: \mathscr{Y}^{<\omega} \rightarrow \mathscr{Y}$. Then $g(c \mid n)=c(n)$ implies $f(\phi(c) \mid n)=\phi(c)(n)$ for $c \in \mathscr{Y}^{\omega}$, and hence $g$ is a winning strategy for the second player in $\Gamma_{\mathscr{y}}^{*}\left(\phi^{-1}[A]\right)$.

(b) Let $\pi: \omega \rightarrow \omega^{<\omega}$ be a canonical bijection. Put $\psi^{\prime}(s)=\pi(s(0))^{\wedge} \pi(s(1))^{\wedge}$ $\ldots \wedge \pi(s(n))$ for $s \in \omega^{n+1}$ and $\psi(c)=\bigcup_{n>0} \psi^{\prime}(c \mid n)$ for $c \in \omega^{\omega}$. Obviously, $\psi: \omega^{\omega} \rightarrow \omega^{\omega}$ is continuous. Suppose that $A \in \mathbb{K}\left(\omega^{\omega}\right)$ and let $G=\bigcap_{n \in \omega} G_{n}$ be disjoint from $A$, where each $G_{n}$ is open dense in $\omega^{\omega}$. For $s \in \omega^{<\omega}$ let 
$f(s) \in \omega$ be such that $\left[\psi^{\prime}(s)^{\wedge} \pi(f(s))\right] \subseteq \bigcap_{n<l h s} G_{n}$. Then $\psi(c) \in G$ provided

$$
(\forall m)(\exists n>m)(f(c \mid n)=c(n)),
$$

and hence $f$ is a winning strategy for Player II in $\Gamma_{\omega}^{*}\left(\psi^{-1}[A]\right)$.

\section{THE NOTION OF FORCING DETERMINED BY $\mathfrak{D}_{\mathscr{X}}$}

Let us consider the factor Boolean algebra $\operatorname{BOREL}\left(\mathscr{Q}^{\omega}\right) / \mathfrak{D}_{\mathscr{Z}}$ as a notion of forcing.

Let $\mathbb{D}_{\mathscr{X}}$ be the family of all trees $T \subseteq \mathscr{X}^{<\omega}$ satisfying $(* *)$ and such that for every $s \in T$ either $\operatorname{succ}_{T}(s)=\mathscr{Z}$ or $\left|\operatorname{succ}_{T}(s)\right|=1$. It is ordered by inclusion. By Theorem 1.2 the order $\mathbb{D}_{\mathscr{L}}$ can be densely embedded in BOREL $(\mathscr{Z} \omega) / \mathfrak{D}_{\mathscr{X}}$; hence they have the same forcing properties.

Note that $\mathbb{D}_{2}$ is the Sacks perfect set forcing.

We define orders $\leq_{n}$ on $\mathbb{D}_{\mathscr{X}}$ considering two cases: $\mathscr{X}$ is finite and $\mathscr{Z}$ is infinite.

Suppose first that $\mathscr{X}=k \in \omega$. For a tree $T \in \mathbb{D}_{k}$ let $T^{n}$ contain all elements $s$ of $T$ such that $\operatorname{succ}_{T}(s)=k$ and there exist exactly $n-1$ initial segments of $s$ with the same property. We write $T_{1} \leq_{n} T_{2}$ if and only if $T_{1} \leq T_{2}$ and $\left(T_{1}\right)^{n}=\left(T_{2}\right)^{n}$.

Now consider the case $\mathscr{X}=\omega$. Fix an enumeration $\left\{s_{n}: n \in \omega\right\}=\omega^{<\omega}$ such that $s_{i} \subseteq s_{j}$ implies $i \leq j$ and such that $s_{i}=s^{\wedge} n, s_{j}=s^{\wedge} m, n<m$, imply $i<j$. Let $T \in \mathbb{D}_{\omega}$. Each element of $\omega^{<\omega}$ determines in a canonical way an element of $\left\{s \in T: \operatorname{succ}_{T}(s)=\omega\right\}$; denote by $T(i)$ the element determined by $s_{i}$. For $T, T^{\prime} \in \mathbb{D}_{\mathscr{L}}$ and $n \in \omega$ put $T \leq_{n} T^{\prime}$ if and only if $T \leq T^{\prime}$ and $\{T(i): i \leq n\}=\left\{T^{\prime}(i): i \leq n\right\}$.

Clearly, orders $\leq_{n}$ have the fusion property and witness Baumgartner's Axiom $A$ for $\mathbb{D}_{\mathscr{K}}$ (cf. [B]).

Let $\mathbb{D}_{\mathscr{L}}=\left\{A \subseteq \omega^{\omega}:\left(\forall T \in \mathbb{D}_{\mathscr{X}}\right)\left(\exists T^{\prime} \in \mathbb{D}_{\mathscr{L}}, T^{\prime} \leq T\right)\left(\left[T^{\prime}\right] \cap A=\varnothing\right)\right\}$. Clearly it is a $\sigma$-ideal containing $\mathfrak{D}_{\mathscr{X}}$. Note that the formula " $c \in \omega^{\omega}$ is a code for a Borel set from $\mathfrak{D}_{\mathscr{X}}$ " is absolute.

Theorem 2.1. (a) $\mathbb{D}_{\omega}$ adds a Cohen real.

(b) $\mathbb{D}_{\omega} \Vdash$ " $2^{\omega} \cap V \notin \mathbb{K}$." Consequently, $\mathbb{D}_{\omega}$ adds neither random reals nor dominating reals.

Proof. (a) Let $G \subseteq \mathbb{D}_{\omega}$ be a generic set over $V, c_{G}=\bigcup\{\operatorname{root} T: T \in G\}$. Obviously $c_{G}: \omega \rightarrow \omega$. Since $\mathfrak{D}_{\omega} \subseteq \mathbb{D}_{\omega}, c_{G} \notin A$ provided $A \in \mathfrak{D}_{\omega}$ is a Borel set coded in $V$. Let $\psi$ be a function given by Proposition 1.3(b) We claim that $\psi\left(c_{G}\right)$ is a Cohen real over $V$. Indeed, if $A \in \mathbb{K}\left(2^{\omega}\right)$ is a Borel set coded in $V$, then $\psi^{-1}[A] \in D_{\omega}$ is a Borel set coded in $V$, too. Hence $c_{G} \notin \psi^{-1}[A]$.

(b) Assume that $T \Vdash$ " $\dot{G}_{n}$ are decreasing open dense subsets of $2^{\omega}$ for $n \in \omega$." Construct inductively sequences $\left\langle T_{n}: n \in \omega\right\rangle,\left\langle S_{n}: n \in \omega\right\rangle \subseteq \mathbb{D}_{\omega}$, $\left\langle v_{n}: n \in \omega\right\rangle \subseteq 2^{<\omega}$, and $\left\langle u_{s}: s \in \omega^{<\omega}\right\rangle \subseteq \omega^{<\omega}$ such that

(0) $u_{s_{0}}=\operatorname{root} T, S_{0} \leq T \mid u_{s_{0}}$, and $v_{0} \in 2^{<\omega}$ satisfy $S_{0} \Vdash$ “ $\left[v_{0}\right] \subseteq \dot{G}_{0}$," $T_{0}$ is a modification of $T$, which below $u_{s_{0}}$ looks like $S_{0}$;

(k) $u_{s_{k} \wedge n}=\operatorname{root} S_{k} \wedge n$ (note that at this moment we have surely defined $\left.u_{s_{k+1}} \in T\right), S_{k+1} \leq T_{k} \mid u_{s_{k+1}}$, and $v_{k+1} \in 2^{<\omega}$ satisfy

$$
S_{k+1} \Vdash \text { “ }\left[v_{0}^{\wedge} \ldots v_{k}^{\wedge} v_{k+1}\right] \subseteq \dot{G}_{k+1}, "
$$


$T_{k+1}$ is a modification of $T_{k}$, which below $u_{s_{k+1}}$ looks like $S_{k+1}$.

Finally note that the sequence $\left\langle u_{s}: s \in \omega^{<\omega}\right\rangle$ determines a condition $T^{\prime} \leq$ $T$, and the sequence $\left\langle v_{n}: n \in \omega\right\rangle$ gives a function $c=v_{0} \wedge v_{1} \wedge v_{2} \wedge \cdots$ from $2^{\omega} \cap V$ such that $T^{\prime} \Vdash$ " $c \in \bigcap_{n \in \omega} G_{n}$."

Remark. Theorem 2.1 shows that, in some sense, $\mathbb{D}_{\omega}$ is close to the Cohen forcing. Actually, we have proved there that if $r \in \omega^{\omega}$ is a real that does not belong to any set from $\mathfrak{D}_{\omega}$ coded in $V$ then there is a Cohen real in $V[r]$. Similarly, one can prove that if reals from the ground model $V$ form a set of the first category in an extension, then $\omega^{\omega} \cap V$ belongs to $\mathfrak{D}_{\omega}$ in this extension. It seems that the forcing $\mathbb{D}_{\omega}$ is the best one for adding Cohen reals in countable support iterations.

Proposition 2.2. Suppose $\mathscr{X}$ if finite. Then

(a) If $T \Vdash$ “ $\tau \in V$," $n \in \omega$ then there are $T^{\prime} \leq_{n} T$ and $\left.A \in[V]^{\mid \mathscr{Q}}\right|^{n}$ such that $T^{\prime} \Vdash$ " $\dot{\tau} \in A$ ";

(b) $\mathbb{D}_{\mathscr{X}} \Vdash$ " $(\forall A \in \mathbb{L})(\exists B \in \mathbb{L} \cap V)(A \subseteq B)$," so the forcing with $\mathbb{D}_{\mathscr{X}}$ does not change the ideal of measure and the ideal of category.

Proof. (b) Applying (a) and the fusion property of orders $\leq_{n}$ one can get that $\mathbb{D}_{\mathscr{X}} \Vdash$ “ $\left(\forall f \in \omega^{\omega}\right)\left(\exists \phi: \omega \rightarrow[\omega]^{<\omega}, \phi \in V\right)(\forall n \in \omega)\left(|\phi(n)|=2^{n} \& f(n) \in\right.$ $\phi(n))$." Hence $\mathbb{D}_{\mathscr{Z}} \Vdash$ "every set of measure zero can be covered by a Borel set of measure zero coded in $V$ "; this implies the analogous property for the ideal $\mathbb{K}, \mathbb{D}_{\mathscr{Q}} \Vdash$ " $(\forall A \in \mathbb{K})(\exists B \in \mathbb{K} \cap V)(A \subseteq B)$ " (cf. [F]).

The above result can be strengthened. Let $k \geq 2$ be a natural number. A tree $T \subseteq \omega^{<\omega}$ is said to be a $k$-tree if $(\forall s \in T)\left(\left|\operatorname{succ}_{T}(s)\right| \leq k\right)$. We say that a notion of forcing $\mathbb{P}$ has the $k$-localization property if

$$
\mathbb{P} \Vdash\left(\forall f \in \omega^{\omega}\right)(\exists T \in V)(\text { “ } T \text { is a } k \text {-tree on } \omega \text { " \& } f \in[T]) .
$$

Let us remark that if $\mathbb{P}$ has the $k$-localization property then, in $V^{\mathbb{P}}$, reals from $(k+1)^{\omega}$ can be covered by sets from $\mathfrak{D}_{k+1}$ coded in $V$. Let $\mathbb{S}_{k}$ be the Silver forcing below $k$. It contains all partial functions $p: \operatorname{dom} p \rightarrow k$ such that $\operatorname{dom} p \subseteq \omega$ is coinfinite and it is ordered by inclusion.

Theorem 2.3. Countable support iterations and countable support products of forcings $\mathbb{D}_{i}$ or $\mathbb{S}_{i}$ for $i \leq k$ have the $k$-localization property.

Proof. We do not find a general proof of this theorem but the proofs of all possible cases are very similar. We present the proof of Theorem 2.3 for countable support product of $\mathbb{D}_{k}$ because we will use this version.

Recall that $T_{1} \leq_{n} T_{2}$ if and only if $T_{1} \leq T_{2}$ and $\left(T_{1}\right)^{n}=\left(T_{2}\right)^{n}$, where $T^{n}$ consists of all elements of $s$ of $T$ such that $\operatorname{succ}_{T}(s)=k$ and there exists exactly $n-1$ initial segments of $s$ with the same property. Note that $\left|T^{n}\right|=k^{n-1}$ for each $n \geq 1$ and $T \in \mathbb{D}_{k}$. Moreover each function $\sigma \in k^{n}$ uniquely determines an element $s=s_{\sigma} \wedge \sigma(n-1)$, where $s_{\sigma} \in T^{n}$. We put $(T)_{\sigma}=T_{s}$.

Let $\mathbb{P}$ be the countable support product of $\kappa$ copies of $\mathbb{D}_{k}$.

For $F \in[\kappa]^{<\omega}$ and $\eta: F \rightarrow \omega$ we define orders $\leq_{F, \eta}$ on $\mathbb{P}$ by $p \leq_{F, \eta} q$ if and only if $p \leq q$ and $(\forall \alpha \in F)\left(p(\alpha) \leq_{\eta(\alpha)} q(\alpha)\right)$. 
Orders $\leq_{F, \eta}$ have the fusion property: Suppose that $p_{n+1} \leq_{F_{n}, \eta_{n}} p_{n}, F_{n} \subseteq$ $F_{n+1}, \bigcup_{n \in \omega} F_{n}=\bigcup_{n \in \omega} \operatorname{supp}\left(p_{n}\right)$ and if $\alpha \in F_{n}$ then

$$
(\forall m \geq n)\left(\eta_{m}(\alpha) \leq \eta_{m+1}(\alpha)\right) \text { and } \lim _{m \rightarrow \infty} \eta_{m}(\alpha)=\infty .
$$

Then there exists $p \in \mathbb{P}$ such that $p \leq_{F_{n}, \eta_{n}} p_{n}$ for every $n \in \omega$.

Let $\sigma \in \prod_{\alpha \in F} k^{\eta(\alpha)}, F \in[\kappa]^{<\omega}$, and $\eta: F \rightarrow \omega$. Let $p \in \mathbb{P}_{\kappa}$. The condition $p^{*} \sigma$ is defined by $\left(p^{*} \sigma\right)(\alpha)=p(\alpha)$ for $\alpha \notin F$ and if $\alpha \in F$ then $\left(p^{*} \sigma\right)(\alpha)=$ $(p(\alpha))_{\sigma(\alpha)}$. Note that $q \leq_{F, \eta} p$ imples $q^{*} \sigma \leq p^{*} \sigma$. Moreover if $p \Vdash$ " $i \in \omega$ " then there exists $q \leq_{F, \eta} p$ such that $q^{*} \sigma$ decides $\dot{\tau}$.

Suppose that $\Vdash$ " $\dot{f} \in \omega^{\omega}$." Let $A \subseteq \omega^{<m}$ be a $k$-tree. We say that $A$ is $(p, F, \eta, \dot{f})$-saturated if

(i) $p \Vdash$ " $\dot{f} \mid m \in A$,"

(ii) $p^{*} \sigma$ decides $\dot{f} \mid m$ for every $\sigma \in \prod_{\alpha \in F} k^{\eta(\alpha)}$,

(iii) if $q \leq_{F, \eta} p, m^{\prime}>m$, and $\sigma_{1}, \sigma_{2} \in \prod_{\alpha \in F} k^{\eta(\alpha)}$ are such that $q^{*} \sigma_{1}$ and $q^{*} \sigma_{2}$ decide $\dot{f} \mid m^{\prime}$ in different ways then they decide $\dot{f} \mid m$ differently.

Claim. Suppose that we have given $p \in \mathbb{P}, F \in[\kappa]^{<\omega}, \eta: F \rightarrow \omega$, and a $(p, F, \eta, f)$-saturated $k$-tree $A \subseteq \omega \leq m$. Let $F_{1}=F \cup\{\beta\}, \eta_{1}=\eta \cup\{(\beta, 1)\}$. Then there exists $q \leq F_{1}, \eta_{1} p$ and a $\left(q, F_{1}, \eta_{1}, \dot{f}\right)$-saturated $k$-tree $A^{\prime} \subseteq \omega \leq M$ containing $A$.

Fix an enumeration $\left\{\sigma_{1}, \ldots, \sigma_{z}\right\}=\prod_{\alpha \in F} k^{\eta(\alpha)}$. Let $\sigma_{i}^{\wedge} j=\sigma_{i} \cup\{(\beta,\langle j\rangle)\}$. We construct conditions $p_{i}$, natural numbers $m_{i}$, and $A^{i} \in\left[\omega^{<\omega}\right]^{\leq k}$ for $i \leq z$ :

$p_{1} \leq_{F_{1}, \eta_{1}} p$ and $m_{1}>m$ are such that $p_{1}^{*}\left(\sigma_{1} \wedge j\right)$ decides $\dot{f} \mid\left[m, m_{1}\right)$ for each $j<k$ and the set $A^{1}=\left\{s \in \omega^{\left[m, m_{1}\right)}:(\exists j<k)\left(p_{1}^{*}\left(\sigma_{1}^{\wedge} j\right) \Vdash\right.\right.$ “ $\left.\dot{f} \mid\left[m, m_{1}\right)=s^{\prime}\right\}$ has the greatest possible cardinality.

$p_{i+1} \leq_{F_{1}, \eta_{1}} p_{i}$ and $m_{i+1}>m_{i}$ are such that $p_{i+1}^{*}\left(\sigma_{l}^{\wedge} j\right)$ decides $\dot{f} \mid\left[m, m_{i+1}\right)$ for each $j<k, l \leq i+1$ and the set $A^{i+1}=\left\{s \in \omega^{\left[m, m_{i+1}\right)}:(\exists j<\right.$ $k)\left(p_{i+1}^{*}\left(\sigma_{i+1}^{\wedge} j\right) \Vdash\right.$ “ $\left.\dot{f} \mid\left[m, m_{i+1}\right)=s "\right\}$ has the greatest possible cardinality.

Note that if $p_{i}^{*}\left(\sigma_{i}^{\wedge} j\right), p_{i}^{*}\left(\sigma_{i}, \wedge j\right)$ decide $\dot{f} \mid m$ in the same way for some $i^{\prime}<i \leq z$ and $j<k$ then they do the same with $\dot{f} \mid m_{1}$. Put $M=m_{z}$, $q=p_{z}$, and $A^{\prime}=\left\{s \in \omega^{M}:(\exists i \leq z, j<k)\left(q^{*}\left(\sigma_{i}^{\wedge} j\right) \Vdash “ \dot{f} \mid M=s "\right)\right\}$. Then $A^{\prime}$ contains $A$ and it is a $k$-tree (by the last remark). To show that it is $\left(q, F_{1}, \eta_{1}, \dot{f}\right)$-saturated we have to verify condition (iii) only. Suppose that $r \leq_{F_{1}, \eta_{1}} q, M^{\prime}>M$, and $\sigma, \delta \in \prod_{\alpha \in F} k^{\eta(\alpha)}, i, j<k$, are such that $r^{*}\left(\sigma^{\wedge} i\right)$ and $r^{*}\left(\delta^{\wedge} j\right)$ decide $\dot{f} \mid M^{\prime}$ in different ways but they decide $\dot{f} \mid M$ in the same way. We may assume that $r^{*}\left(\sigma^{\wedge} j\right)$ and $r^{*}\left(\delta^{\wedge} i\right)$ decide $\dot{f} \mid M^{\prime}$. Then $r^{*}\left(\sigma^{\wedge} i\right)$ and $r^{*}\left(\delta^{\wedge} i\right)$ decide $\dot{f} \mid M^{\prime}$ in the same way. Hence $r^{*}\left(\delta^{\wedge} i\right)$ and $r^{*}\left(\delta^{\wedge} j\right)$ decide $\dot{f} \mid M^{\prime}$ differently. But it follows from the construction (maximality of $A^{i}$ ) that then they decide $\dot{f} \mid M$ differently-a contradiction.

Claim. Suppose that we have given $p \in \mathbb{P}, F \in[\kappa]^{<\omega}, \eta: F \rightarrow \omega$, and a $(p, F, \eta, \dot{f})$-saturated $k$-tree $A \subseteq \omega^{\leq m}$. Let $\beta \in F$ and $\eta_{1}$ be a modification of $\eta$ such that $\eta_{1}(\beta)=\eta(\beta)+1$. Then there exist $q \leq_{F, \eta_{1}} p$ and a $\left(q, F, \eta_{1}, \dot{f}\right)$-saturated $k$-tree $A^{\prime} \subseteq \omega \leq M$ containing $A$.

The proof of this claim can be obtained from the proof of the previous one by redefining $\sigma^{\wedge} j$. Now it should be equal $\sigma \mid F \backslash\{\beta\} \cup\left\{\left\langle\beta, \sigma(\beta)^{\wedge} j\right\rangle\right\}$. 
Suppose $p \Vdash$ “ $\dot{f} \in \omega^{\omega}$." Apply the claims above to construct $p_{n} \leq p, F_{n} \in$ $[\kappa]^{<\omega}, \eta_{n}: F_{n} \rightarrow \omega$ with the fusion property, and increasing $k$-trees $A_{n} \subseteq$ $\omega^{\leq m} n$ such that $m_{n}<m_{n+1}$ and $p_{n} \Vdash$ " $\dot{f} \mid m_{n} \in A_{n}$." Let $A=\bigcup_{n \in \omega} A_{n}$ and let $q$ be the fusion of $\left\langle p_{n}: n \in \omega\right\rangle$. Then $A$ is a $k$-tree and $q \Vdash$ " $\dot{f} \in[A]$."

Since obviously forcings $\mathbb{D}_{k+1}$ and $\mathbb{S}_{k+1}$ do not have the $k$-localization property forcings with this property, add neither $\mathbb{D}_{k+1}$ nor $\mathbb{S}_{k+1}$-generic reals. Thus we get

Corollary 2.4. No countable support iteration of forcings $\mathbb{S}_{2}$ adds an $\mathbb{S}_{4}$-generic real.

\section{CARDinal COEFFICIENTS}

For an ideal $\mathbb{I}$ of subsets of a space $X$ the following cardinal invariants of II, we introduce

$$
\begin{aligned}
& \operatorname{add}(\mathbb{I})=\min \{|\mathscr{H}|: \mathscr{H} \subseteq \mathbb{I} \& \cup \mathscr{H} \notin \mathbb{I}\} \\
& \operatorname{cov}(\mathbb{I})=\min \{|\mathscr{H}|: \mathscr{H} \subseteq \mathbb{I} \& \bigcup \mathscr{H}=X\} \\
& \operatorname{non}(\mathbb{I})=\min \{|H|: H \subseteq X \& H \notin \mathbb{I}\} \\
& \operatorname{cof}(\mathbb{I})=\min \{|\mathscr{H}|: \mathscr{H} \subseteq \mathbb{I} \&(\forall A \in \mathbb{I})(\exists B \in \mathscr{H})(A \subseteq B)\}
\end{aligned}
$$

The cardinal coefficients of ideals $\mathbb{L}\left(2^{\omega}\right)$ and $\mathbb{K}\left(2^{\omega}\right)$, together with the unbounded number $\mathfrak{b}$ and the dominating number $\mathfrak{d}$, are collected in the so-called Cichoń Diagram (cf. [F, V]).

Theorem 3.1. (a) $\operatorname{cov}\left(\mathfrak{D}_{\mathscr{X}}\right) \geq \operatorname{cov}\left(\mathfrak{D}_{\mathscr{Y}}\right)$ and non $\left(\mathfrak{D}_{\mathscr{X}}\right) \leq \operatorname{non}\left(\mathfrak{D}_{\mathscr{Y}}\right)$, provided $\mathscr{X} \subseteq \mathscr{Y}$.

(b) If $\mathscr{X}$ is finite then $\operatorname{cov}\left(\mathfrak{D}_{\mathscr{X}}\right) \geq \max \{\operatorname{cov}(\mathbb{K}), \operatorname{cov}(\mathbb{L})\}$ and $\operatorname{non}\left(\mathfrak{D}_{\mathscr{X}}\right) \leq$ $\min \{\operatorname{non}(\mathbb{K}), \operatorname{non}(\mathbb{L})\}$.

(c) $\operatorname{cov}\left(\mathfrak{D}_{\omega}\right)=\operatorname{cov}(\mathbb{K})$ and $\operatorname{non}\left(\mathfrak{D}_{\omega}\right)=\operatorname{non}(\mathbb{K})$.

Proof. (a) Let $\phi: \mathscr{Y}^{\omega} \rightarrow \mathscr{X}^{\omega}$ be a function given by Proposition 1.3(a). Then $\phi[A] \notin \mathfrak{D}_{\mathscr{Z}}$ provided $A \notin \mathfrak{D}_{\mathscr{Y}}$. If $A_{\alpha} \in \mathfrak{D}_{\mathscr{X}}(\alpha<\kappa), \bigcup_{\alpha<\kappa} A_{\alpha}=\mathscr{X}^{\omega}$ then $\phi^{-1}\left[A_{\alpha}\right] \in \mathfrak{D}_{\mathscr{y}}$ and $\bigcup_{\alpha<\kappa} \phi^{-1}\left[A_{\alpha}\right]=\mathscr{Y}^{\omega}$.

(b) follows from Theorem 1.1(b).

(c) was proved in [R1], where Bartoszyński's characterization of non $(\mathbb{K})$ and $\operatorname{cov}(\mathbb{K})$ was applied. Note, however, that we can apply Proposition 1.3(b) to prove, similarly as in $(a)$, that $\operatorname{non}\left(\mathfrak{D}_{\omega}\right) \geq \operatorname{non}(\mathbb{K})$ and $\operatorname{cov}\left(\mathfrak{D}_{\omega}\right) \leq \operatorname{cov}(\mathbb{K})$. Converse inequalities follows from Theorem 1.1(b).

Theorem 3.2. There exists a family $F \subseteq \mathfrak{D}_{\mathscr{Z}}$ of the cardinality $\mathfrak{c}$ such that for every uncountable $G \subseteq F, \bigcup G \notin \mathfrak{D}_{\mathscr{X}}$.

Proof. Let $\left\{c_{\alpha}: \alpha<\mathfrak{c}\right\} \subseteq \mathscr{X}^{\omega}$ be such a family that $\alpha<\beta<\mathfrak{c}$ implies $(\forall n)(\exists m>n)\left(c_{\alpha}(m) \neq c_{\beta}(m)\right)$. For $\alpha<\mathfrak{c}$ put $A_{\alpha}=\left\{c \in \mathscr{X}^{\omega}:(\forall n)(c(n) \neq\right.$ $\left.\left.c_{\alpha}(n)\right)\right\}$. Clearly $A_{\alpha} \in \mathfrak{D}_{\mathscr{X}}$. Suppose that $R \subseteq \mathfrak{c}$ is uncountable. We want to show that $\bigcup_{\alpha \in R} A_{\alpha} \notin \mathfrak{D}_{\mathscr{X}}$. Suppose not and let $f: \mathscr{X}<\omega \rightarrow \mathscr{X}$ witness that $\bigcup_{\alpha \in R} A_{\alpha} \in \mathfrak{D}_{\mathscr{X}}$. For $\alpha<\mathfrak{c}$ and $s \in \mathscr{X}^{<\omega}$, let $B_{\alpha}(s)=\left\{t \in \mathscr{X}^{<\omega}: s \subseteq t \&(\forall n \in\right.$ $\left.\operatorname{dom} t \backslash \operatorname{dom} s)\left(t(n) \neq c_{\alpha}(n)\right)\right\}$.

Claim. $(\exists \alpha \in R)\left(\forall s \in \mathscr{X}^{<\omega}\right)\left(\exists t \in B_{\alpha}(s)\right)\left(f(t) \neq c_{\alpha}(\operatorname{lh}(t))\right)$. If not, we would find distinct $\alpha, \beta \in R$ and $s \in \mathscr{X}^{<\omega}$ such that $\left(\forall t \in B_{\alpha}(s)\right)\left(f(t)=c_{\alpha}(\ln (t))\right)$ and $\left(\forall t \in B_{\beta}(s)\right)\left(f(t)=c_{\beta}(\operatorname{lh}(t))\right)$. Let $n \geq \operatorname{lh}(s)$ be the first such that 
$c_{\alpha}(n) \neq c_{\beta}(n)$. Choose $t \in \mathscr{X}^{n}$ such that $s \subseteq t$ and $(\forall k \in n \backslash \operatorname{dom} s)(t(k) \neq$ $\left.c_{\alpha}(k)=c_{\beta}(k)\right)$. Then $t \in B_{\alpha}(s) \cap B_{\beta}(s)$ and hence $c_{\alpha}(n)=f(t)=c_{\beta}(n)-\mathrm{a}$ contradiction.

Thus let $a \in R$ be given by the claim. Construct inductively a sequence $\left\langle t_{n}: n \in \omega\right\rangle \subseteq \mathscr{X}^{<\omega}$ such that $t_{0} \in B_{\alpha}(\varnothing)$ and $f\left(t_{0}\right) \neq c_{\alpha}\left(\ln \left(t_{0}\right)\right), t_{n+1} \in$ $B_{\alpha}\left(t_{n} \wedge f\left(t_{n}\right)\right)$, and $f\left(t_{n+1}\right) \neq c_{\alpha}\left(\operatorname{lh}\left(t_{n+1}\right)\right)$. Put $d=\bigcup_{n \in \omega} t_{n}$. Then evidently $d \in A_{\alpha} \backslash\left\{c \in \mathscr{X}^{\omega}:(\exists n \in \omega)(\forall m>n)(c(m) \neq f(c \mid m))\right\}$, and hence $f$ cannot witness that $\bigcup_{\alpha \in R} A_{\alpha} \in \mathfrak{D}_{\mathscr{X}}$.

Corollary 3.3. $\operatorname{add}\left(\mathfrak{D}_{\mathscr{X}}\right)=\omega_{1}$ and $\operatorname{cof}\left(\mathfrak{D}_{\mathscr{X}}\right)=2^{\omega}$.

Putting together Corollary 3.3 and Theorems 3.1 (c) we get the full description of the cardinal coefficients of $\mathfrak{D}_{\omega}: \operatorname{add}\left(\mathfrak{D}_{\omega}\right)=\omega_{1}, \operatorname{non}\left(\mathfrak{D}_{\omega}\right)=\operatorname{non}(\mathbb{K})$, $\operatorname{cov}\left(\mathfrak{D}_{\omega}\right)=\operatorname{cov}(\mathbb{K})$, and $\operatorname{cof}\left(\mathfrak{D}_{\omega}\right)=\mathfrak{c}$. Below we give some consistency results concerning the coefficients non and cov of $\mathfrak{D}_{\mathscr{X}}$ for finite $\mathscr{X}$.

Theorem-3.4. (a) $\operatorname{CON}\left(Z F C+(\forall k \geq 2)\left(\operatorname{cov}\left(\mathfrak{D}_{k}\right)=\omega_{2}=\mathfrak{c}\right)+\operatorname{cof}(\mathbb{L})=\omega_{1}\right)$.

(b) Suppose that $k \geq 2$. Then $\operatorname{CON}\left(\mathrm{ZFC}+\operatorname{cov}\left(\mathfrak{D}_{k+1}\right)=\operatorname{cof}(\mathbb{L})=\omega_{1}+\right.$ $\left.\operatorname{cov}\left(\mathfrak{D}_{k}\right)=\omega_{2}=\mathfrak{c}\right)$.

Proof. (a) Suppose that $V \Vdash \mathrm{CH}$. Let $\mathbb{P}=\prod_{\alpha<\kappa} \mathbb{P}_{\alpha}$ be the countable support product of forcings $\mathbb{D}_{k}(k \geq 2)$ such that $\left|\left\{\alpha<\kappa: \mathbb{P}_{\alpha}=\mathbb{D}_{k}\right\}\right|=\omega_{2}$ for each $k \geq 2$. Standard arguments (see [B]) show that $\mathbb{P}$ preserves cardinal numbers and $\mathbb{P} \Vdash$ "c $=\omega_{2}$." Moreover, if $F \in\left[\omega_{2}\right]^{\leq n}$ is such that for $\alpha \in F, \mathbb{P}_{\alpha}=\mathbb{D}_{m}$ for some $m \leq n$ and $p \Vdash$ " $\dot{\tau} \in \omega$ " then there exist $q \leq_{F, n} p$ and $A \subseteq \omega,|A| \leq$ $n^{n \cdot n}$ such that $q \Vdash$ " $\dot{\tau} \in A$ ". Hence by fusion arguments one can prove that $\mathbb{P} \vdash$ " $\left(\forall f \in \omega^{\omega}\right)\left(\exists \phi: \omega \rightarrow[\omega]^{<\omega}, \phi \in V\right)(\forall n \in \omega)\left(f(n) \in \phi(n) \&|\phi(n)| \leq n^{n \cdot n}\right) "$ and consequently $\mathbb{P} \Vdash$ " $(\forall A \in \mathbb{L})(\exists B \in \mathbb{L} \cap V)(A \subseteq B)$." Hence $\mathbb{P} \Vdash$ " $\operatorname{cof}(\mathbb{L})=$ $\omega_{1}$ " (which in particular, means that $\Vdash_{\omega_{2}}$ " non $\left(\mathfrak{D}_{\mathscr{X}}\right)=\omega_{1}$ " for each finite $\mathscr{X}$ ). Let $k \geq 2$.

Claim. $\mathbb{P} \Vdash “ \operatorname{cov}\left(\mathfrak{D}_{k}\right)=\omega_{2} . "$

First note that if $f: k^{<\omega} \rightarrow k$ and $c$ is a $\left(\mathbb{D}_{k}\right)^{V}$-generic real over $V[f]$ then infinitely often $f(c \mid m)=c(m)$. Let $\left\langle c_{\alpha}: \alpha<\kappa\right\rangle$ be a $\mathbb{P}$-generic sequence over $V$. Suppose now that, in $V\left[\left\langle c_{\alpha}: \alpha<\kappa\right\rangle\right]$, for each $\alpha<\omega_{1}$ we have given a function $f_{\alpha}: k^{<\omega} \rightarrow k$. Let $\dot{f}_{\alpha}$ be a $\mathbb{P}$-name for $f_{\alpha}$. Each $\dot{f}_{\alpha}$ is a $\prod_{\beta<\beta(\alpha)} \mathbb{P}_{\beta^{-}}$ name for some $\beta(\alpha)<\omega_{2}$. Choose $\beta<\omega_{2}$ such that $\mathbb{P}_{\beta}=\mathbb{D}_{k}$ and $\beta(\alpha)<\beta$ for each $\alpha<\omega_{1}$. Thus $\left\langle\dot{f}_{\alpha}: \alpha<\omega_{1}\right\rangle$ is a sequence $\prod_{\alpha<\beta} \mathbb{P}_{\alpha}$-names. Since $c_{\beta}$ is $\mathbb{D}_{k}$-generic over $V\left[\left\langle c_{\alpha}: \alpha<\beta\right\rangle\right]$ we get that, in $V\left[\left\langle c_{\alpha}: \alpha<\kappa\right\rangle\right]$, for each $\alpha<\omega_{1}$ infinitely often $f_{\alpha}\left(c_{\beta} \mid m\right)=c_{\beta}(m)$. Consequently, sets from $\mathfrak{D}_{k}$ determined in $V\left[\left\langle c_{\alpha}: \alpha<\kappa\right\rangle\right]$ by functions $f_{\alpha}$ do not cover $k^{\omega}$.

(b) Fix $k \geq 2$. Suppose $V \vDash C H$. Let $\mathbb{P}$ be a countable support product of $\omega_{2}$ copies of forcings $\mathbb{D}_{k}$. Then $\mathbb{P}$ preserves cardinal numbers and $\mathbb{P} \Vdash$ “ $\operatorname{cof}(\mathbb{L})=\omega_{1} \& \mathfrak{c}=\omega_{2}$." By Theorem 2.3, $\mathbb{P}$ has the $k$-localization property. Thus, in $V^{\mathbb{P}},(k+1)^{\omega}$ can be covered by sets from $\mathfrak{D}_{k+1}$ coded in $V$. Consequently $\mathbb{P} \Vdash “ \operatorname{cov}\left(\mathfrak{D}_{k+1}\right)=\omega_{1}$." As in (a) one can prove that $\mathbb{P} \Vdash “ \operatorname{cov}\left(\mathfrak{D}_{k}\right)$ $=\omega_{2} . "$

Theorem 3.5. $\operatorname{CON}\left(\mathrm{ZFC}+(\forall k \geq 3)\left(\operatorname{non}\left(\mathfrak{D}_{k}\right)=\operatorname{cov}(\mathbb{K})=\omega_{2}\right)\right)$.

Proof. Let $\mathbb{P}$ consist of all pairs $(T, n)$ such that $T$ is a 2 -tree on $\omega,|[T]|<$ $\omega$, and $\left|\operatorname{succ}_{T}(s)\right|=1$ for each $s \in T$ of length $\geq n$. We order $\mathbb{P}$ by $(T, n) \leq(S, m)$ if and only if $S \subseteq T, n \geq m$, and $T|m=S| m$. 
Claim. $\mathbb{P}$ has the ccc property.

Suppose that $\left(T_{\alpha}, n_{\alpha}\right) \in \mathbb{P}$ for $\alpha<\omega_{1}$. We may assume that $n_{\alpha}=n$ and $T_{\alpha} \mid n=S$ for every $\alpha<\omega_{1}$. Let $\alpha<\beta<\omega_{1}$. Put $T=T_{\alpha} \cup T_{\beta}$. Then $T \mid n=S$ and $s \in T, \ln (s) \geq n$ imply that $s$ has exactly one successor in each $T_{\alpha}$ and $T_{\beta}$. Hence $T$ is a 2-tree, $|[T]|<\omega$. Choose $m>n$ such that for each $s \in T, \operatorname{lh}(s) \geq m$ there is one successor of $s$ in $T$ only. Then $(T, m) \in \mathbb{P}$ and it lies below $\left(T_{\alpha}, n\right)$ and $\left(T_{\beta}, n\right)$.

Claim. $\mathbb{P} \Vdash$ " $\omega^{\omega} \cap V$ can be covered by countably many 2-trees on $\omega$ " and consequently $\mathbb{P} \Vdash$ “ $(\forall k \geq 3)\left(k^{\omega} \cap V \in \mathfrak{D}_{k}\right)$."

Let $G \subseteq \mathbb{P}$ be a generic set over $V, T_{G}=\bigcup\{T:(\exists n)((T, n) \in G)\}$. Clearly $T_{G}$ is a 2-tree on $\omega$. For $s \in \omega^{n}$ and $t \in T_{G} \cap \omega^{n}$, put $T_{s, t}=\left\{r \in \omega^{<\omega}: r \subseteq s\right.$ or $\left.\left(\exists t^{\prime} \in \omega^{<\omega}\right)\left(r=s^{\wedge} t^{\prime} \& t^{\wedge} t^{\prime} \in T\right)\right\}$. Obviously $T_{s, t}$ are 2-trees. Easy density arguments show that, in $V[G], \omega^{\omega} \cap V \subseteq \bigcup\left\{\left[T_{s, t}\right]: n \in \omega, s \in \omega^{n}, t \in T_{G} \cap\right.$ $\left.\omega^{n}\right\}$.

Suppose that $V \models \mathrm{CH}$. Let $\left\langle\mathbb{P}_{\alpha}, \dot{\mathbb{Q}}_{\alpha}: \alpha<\omega_{2}\right\rangle$ be a finite support iteration of forcings $\mathbb{P}$. Then $\mathbb{P}_{\omega_{2}}$ has the ccc property and $\Vdash_{\omega_{2}}$ "for each $\alpha<\omega_{2}$, $\omega^{\omega} \cap V^{\mathbf{P}} \alpha$ can be covered by countably many 2-trees on $\omega$." Since, in $V^{\mathbb{P}_{\omega}} 2$, each subset of $\omega^{\omega}$ of the cardinality $\omega_{1}$ is included in $\omega^{\omega} \cap V^{\mathbb{P}} \alpha$ for some $\alpha<\omega_{2}$ we get $\Vdash_{\omega_{2}}$ " $(\forall k \geq 3)\left(\operatorname{non}\left(\mathfrak{D}_{k}\right)=\omega_{2}\right)$." Because of Cohen reals added at limit stages we have $\Vdash_{\omega_{2}} " \operatorname{cov}(\mathbb{K})=\omega_{2}$ " (hence $\Vdash_{\omega_{2}} "(\forall k \geq 2)\left(\operatorname{cov}\left(\mathfrak{D}_{k}\right)=\right.$ $\left.\omega_{2}\right)$ ") .

Note that in models presented in Theorems 3.4 and 3.5 we have $\operatorname{cov}\left(\mathfrak{D}_{k}\right) \geq$ $\operatorname{non}\left(\mathfrak{D}_{k}\right)$ for each $k \geq 3$. Thus the following question seems to be natural.

Problem 3.6. Suppose that $\mathscr{Z}$ is finite (but $|\mathscr{Z}| \geq 3$ ). Can non $\left(\mathfrak{D}_{\mathscr{X}}\right)$ be greater than $\operatorname{cov}\left(\mathfrak{D}_{\mathscr{L}}\right)$ ?.

\section{COMPACT SETS FROM THE IDEAL}

Let $\mathscr{K}\left(\mathscr{X}^{\omega}\right)$ denote the space of all compact subsets of $\mathscr{X}^{\omega}$, equipped with the Vietoris topology generated by the subbase consisting of the sets $U(G)=$ $\left\{F \in \mathscr{K}\left(\mathscr{X}^{\omega}\right): F \subseteq G\right\}, V(G)=\left\{F \in \mathscr{K}\left(\mathscr{X}^{\omega}\right): F \cap G \neq \varnothing\right\}$ where $G$ denotes an open subset of $\mathscr{X}^{\omega}$. It is well known that $\mathscr{K}\left(\mathscr{X}^{\omega}\right)$ is a perfect Polish space that is compact if $\mathscr{X}$ is finite (cf. [K]).

Some $\sigma$-ideals have recently been studied from the point of view of the structure of their traces on compact sets. Kechris, Louveau, and Woodin showed that the trace of each $\sigma$-ideal on compact sets is either very simple $\left(\Pi_{2}^{0}\right)$ or at least $\Pi_{1}^{1}$ (cf. [KLW]). Our next result shows that for the ideal $\mathfrak{D}_{\mathscr{L}}$ the second possibility takes place.

Theorem 4.1. Let $\mathscr{X}$ be finite. Then $\mathfrak{D}_{\mathscr{X}} \cap \mathscr{K}\left(\mathscr{X}^{\omega}\right) \in \Pi_{1}^{1} \backslash \Sigma_{1}^{1}$ and it is of the first category in $\mathscr{K}\left(\mathscr{X}^{\omega}\right)$.

Proof. Since $\mathscr{X}^{<\omega}$ is countable the space $\mathscr{P}$ of all subsets of $\mathscr{X}^{<\omega}$ with the obvious product topology is homeomorphic with $2^{\omega}$. Trees form the closed subset of this space. For $A \in \mathscr{K}\left(\mathscr{X}^{\omega}\right)$ we have

$$
A \notin \mathfrak{D}_{\mathscr{Z}} \equiv(\exists T \in \mathscr{P})(\text { “ } T \text { is a tree " \& " } T \text { satisfies }(* *) \text { " \& }[T] \subseteq A) .
$$

The formula " $T$ is a tree and $T$ satisfies $(* *)$ " presents a $\Pi_{2}^{0}$ subset of $\mathscr{P}$. Moreover, $[T] \subseteq A \equiv\left(\forall x \in \mathscr{X}^{\omega}\right)(x \in A \vee(\exists n \in \omega)(x \mid n \notin T))$. The formula 
$x \in A \vee(\exists n \in \omega)(x \mid n \notin T)$ describes a $\Pi_{2}^{0}$ subset of $\mathscr{P} \times \mathscr{K}\left(\mathscr{X}^{\omega}\right) \times \mathscr{X}^{\omega}$. Since $\mathscr{X}^{\omega}$ is compact, the $\forall^{\mathscr{X} \omega}$-projection of a $\Pi_{2}^{0}$ set is of the class $\Pi_{2}^{0}$. Hence the complement of $\mathfrak{D}_{\mathscr{X}} \cap \mathscr{K}\left(\mathscr{X}^{\omega}\right)$ is analytic in $\mathscr{K}\left(\mathscr{X}^{\omega}\right)$. It follows from Theorem 6.4 of [R2] that $\mathfrak{D}_{\mathscr{X}} \cap \mathscr{K}\left(\mathscr{X}^{\omega}\right)$ is of the first category. Since $\mathfrak{D}_{\mathscr{X}} \cap \mathscr{K}\left(\mathscr{X}^{\omega}\right)$ is dense (it contains all finite sets) and it is of the first category, it cannot be $\Pi_{2}^{0}$. Hence $\mathfrak{D}_{\mathscr{X}} \cap \mathscr{K}\left(\mathscr{X}^{\omega}\right) \notin \Sigma_{1}^{1}$ (cf. [KLW]).

For $A \subseteq \mathscr{X}^{\omega} \times \mathscr{X}^{\omega}$ and $d \in \mathscr{X}^{\omega}$, let $A_{d}=\left\{c \in \mathscr{X}^{\omega}:(d, c) \in A\right\}$. Since every set from $\operatorname{BOREL}\left(\mathscr{X}^{\omega}\right) \backslash \mathfrak{D}_{\mathscr{X}}$ contains a compact set that does not belong to $\mathfrak{D}_{\mathscr{X}}$, Theorem 4.1 can be applied to establish the complexity of the class of sets of the form $\left\{c \in \mathscr{X}^{\omega}: A_{c} \notin \mathfrak{D}_{\mathscr{X}}\right\}$. Let $\Phi: \mathscr{P}\left(\mathscr{X}^{\omega} \times \mathscr{X}^{\omega}\right) \rightarrow \mathscr{P}\left(\mathscr{X}^{\omega}\right)$ be given by $\Phi(A)=\left\{c \in \mathscr{X}^{\omega}: A_{c} \notin \mathfrak{D}_{\mathscr{X}}\right\}$. Then easy calculations and an application of Theorem 4.1 show

Corollary 4.2. (a) $\Phi\left[\Sigma_{1}^{0}\left(\mathscr{X}^{\omega} \times \mathscr{X}^{\omega}\right)\right]=\Sigma_{1}^{0}\left(\mathscr{X}^{\omega}\right)$.

(b) $\Phi\left[\Pi_{1}^{0}\left(\mathscr{X}^{\omega} \times \mathscr{X}^{\omega}\right)\right]=\Phi\left[\Sigma_{3}^{0}\left(\mathscr{X}^{\omega} \times \mathscr{X}^{\omega}\right)\right]=\Sigma_{1}^{1}\left(\mathscr{X}^{\omega}\right)$.

(c) $\Phi\left[\operatorname{BOREL}\left(\mathscr{X}^{\omega} \times \mathscr{X}^{\omega}\right)\right] \subseteq \Sigma_{2}^{1}\left(\mathscr{X}^{\omega}\right)$.

\section{REFERENCES}

[B] J. E. Baumgartner, Iterated forcing, Proceedings of the Summer School in Set Theory (A. R. D. Mathias, ed.), Cambridge Univ. Press, Cambridge, 1983.

[D] M. Davis, Infinite games of perfect information, Advances in Game Theory (M. Dresher, L. S. Shapley, and A. W. Tucker, eds.), Ann. of Math. Stud., vol. 52, Princeton Univ. Press, Princeton, NJ, 1964.

[F] D. Fremlin, Cichon's diagram, Seminaire Initiation a l'Analyse (G. Choquet, M. Rogalski, J. Saint Raymond, eds.), 23e annee, 1983/84, no. 5.

[J] T. Jech, Set theory, Academic Press, New York, 1978.

[KLW] A. S. Kechris, A. Louveau, and W. H. Woodin, The structure of $\sigma$-ideals of compact sets, Trans. Amer. Math. Soc. 301 (1987), 263-288.

[M] J. Mycielski, On the axiom of determinateness, Fund. Math. 59 (1966), 203-212.

[R1] A. Rosłanowski, On game ideals, Colloq. Math. 59 (1990), 159-168.

[R2] _ Mycielski ideals generated by uncountable systems, preprint, 1990.

[V] J. E. Vaughan, Small uncountable cardinals and topology, Open Problems in Topology (G. M. Reed and J. van Mill, eds.), North Holland, Amsterdam, 1990.

Mathematical Institute, Polish Academy of Science, ul. Kopernika 18, 51-617 Wroclaw, Poland

Mathematical Institute, University of Wroclaw, Pl. Grunwaldzki $2 / 4,50-384$ Wroclaw, Poland 